\title{
Sellers Characterization in Direct Selling Systems through Data Mining and Analytics
}

Julián Eloy Tornillo, Master ${ }^{1}$, Thomas Gill, Engineer ${ }^{1}$, Mathias Mario Riquelme ${ }^{1}$.

${ }^{1}$ Instituto de Investigaciones en Ingeniería Industrial, Facultad de Ingeniería, Universidad Nacional de Lomas de Zamora, Argentina, jtornillo@ingenieria.unlz.edu.ar, thomas14gill@gmail.com, riquelmemathias5@gmail.com

\begin{abstract}
The direct selling industry presents many opportunities for people who wish to obtain income through the generation of their own business, based on a sales network.

In this business model, direct sellers have objectives that transcend the sales activities themselves, such as establishing sustainable interpersonal relationships with their clients in the medium and long term and abilities in administration and management.

In this work, we study the performance of direct sellers using traditional data in combination with personality traits and personal profiles of sellers through the DISC test. Results are subjected to statistical analysis, using Data Mining techniques and analytics, such as Principal Component Analysis and Clustering.

Results validate those desirable traits for a traditional seller in this industry and show how they are combined with traditional data to identify and describe different groups of behaviour. Besides, we approach the guidelines for an optimal process of sales engineering in this industry.
\end{abstract}

Keywords - Engineering, Data Mining, Direct Selling, Personality Traits

Digital Object Identifier (DOI):

http://dx.doi.org/10.18687/LACCEI2020.1.1.652

ISBN: 978-958-52071-4-1 ISSN: 2414-6390

$18^{\text {th }}$ LACCEI International Multi-Conference for Engineering, Education, and Technology: "Engineering, Integration, and Alliances for a Sustainable Development" "Hemispheric Cooperation for Competitiveness and Prosperity on a Knowledge-Based Economy", July 27-31, 2020, Virtual Edition. 


\title{
Sellers Characterization in Direct Selling Systems through Data Mining and Analytics.
}

\author{
Julián Eloy Tornillo, Master ${ }^{1}$, Thomas Gill, Engineer ${ }^{1}$, Mathias Mario Riquelme ${ }^{1}$. \\ ${ }^{1}$ Instituto de Investigaciones en Ingeniería Industrial, Facultad de Ingeniería, Universidad Nacional de Lomas de Zamora, \\ Argentina, jtornillo@ingenieria.unlz.edu.ar, thomas14gill@gmail.com, riquelmemathias5@gmail.com
}

\begin{abstract}
The direct selling industry presents many opportunities for people who wish to obtain income through the generation of their own business, based on a sales network.

In this business model, direct sellers have objectives that transcend the sales activities themselves, such as establishing sustainable interpersonal relationships with their clients in the medium and long term and abilities in administration and management.

In this work, we study the performance of direct sellers using traditional data in combination with personality traits and personal profiles of sellers through the DISC test. Results are subjected to statistical analysis, using Data Mining techniques and analytics, such as Principal Component Analysis and Clustering.

Results validate those desirable traits for a traditional seller in this industry and show how they are combined with traditional data to identify and describe different groups of behaviour. Besides, we approach the guidelines for an optimal process of sales engineering in this industry.

Keywords- Engineering, Data Mining, Direct Selling, Personality Traits
\end{abstract}

\section{INTRODUCCIÓN}

\section{A. El modelo}

A pesar de la trayectoria y persistencia del modelo de venta directa, el conocimiento público y el desarrollo en investigación que se ha realizado sobre la temática es relativamente reciente. El primer autor que estableció una definición formal fue Baker en 1984, definiendo al modelo de venta directa como una forma de venta sin puntos de venta, distribuidores, mayoristas o cualquier tipo de intermediarios [1]. Más adelante surgieron diversas definiciones que postulaban diferentes visiones de lo que significa este modelo. Según Bennet, el modelo de venta directa es un enfoque de marketing que implica la venta directa de productos y servicios a consumidores a través de explicaciones personales y demostraciones, principalmente en sus casas [2]. Por su parte, Ostrow y Smith definen a este modelo de negocio desde el cómo se realiza la venta, sosteniendo que estas se deben comúnmente a actividades tales como la venta telefónica, venta puerta a puerta o a través de reuniones (parties) en las casas [3]. Ya en la década de los 90' surgen autores que adoptan una visión desde diferentes aristas que se presentan en este tipo de sistemas, y estudian en profundidad su impronta operacional, táctica y estratégica. En este sentido, son entendidos como un método de distribución de bienes y servicios a través de personal que no trabaja en emplazamientos físicos de la compañía, y lo hace generalmente en su casa. Desde el aspecto operacional, la venta directa puede ser tratada como una forma de comunicación interpersonal o de venta personal. Desde una perspectiva táctica la venta directa puede ser considerada como una forma de organizar las actividades de venta y la función de venta. Estratégicamente, la venta directa puede ser vista como un canal o modo de distribución, o una manera de ganar acceso a un mercado para potenciar un negocio [4].

\section{B. Controversia y virtudes}

Existe cierta discusión que pone en juicio el componente ético en los modelos de venta directa. Por un lado, se hace énfasis en que podría ser visto como una precarización del trabajo o una forma de reducción de costos laborales. Se utiliza como argumento que las personas no trabajan directamente para las compañías bajo relación de dependencia, sino que lo hacen de forma independiente y su subsistencia depende exclusivamente de los márgenes obtenidos por sus ventas personales. Contrariamente, se señala que este modelo permite a las personas emprender un negocio propio, y las decisiones de cómo llevar a cabo el mismo. Cada quien establece sus horarios, su método de distribución, si adopta una estrategia de expansión de redes de vendedores o si lo hace mediante la exposición de sus productos en su propio hogar. En esta línea, se la define a la venta directa como un modelo de negocio que presenta oportunidades de desarrollo personal, profesional y económico para todas aquellas personas que deseen obtener ingresos por medio de la generación de un negocio propio [5].

\section{Situación actual}

El modelo de venta directa lleva décadas de crecimiento sostenido tanto en popularidad como en cantidad de ventas. Es en momentos de crisis y recesión económica es cuando muestra su pico de crecimiento en muchas compañías, ya que es una alternativa de generación de ingresos con alto grado de flexibilidad e inversiones iniciales relativamente bajas. Hay más de 100 millones de personas que se dedican a comercializar productos y servicios con esta modalidad, ya sea a tiempo parcial o completo. El rubro que más incidencia tiene bajo esta modalidad de venta es el de productos de belleza y cuidado personal, representando más del $50 \%$ del mercado global del sector, como se muestra en la Figura 1. Además este crecimiento se acentúa más en aquellas economías en vía de desarrollo que en los países desarrollados[6]. 


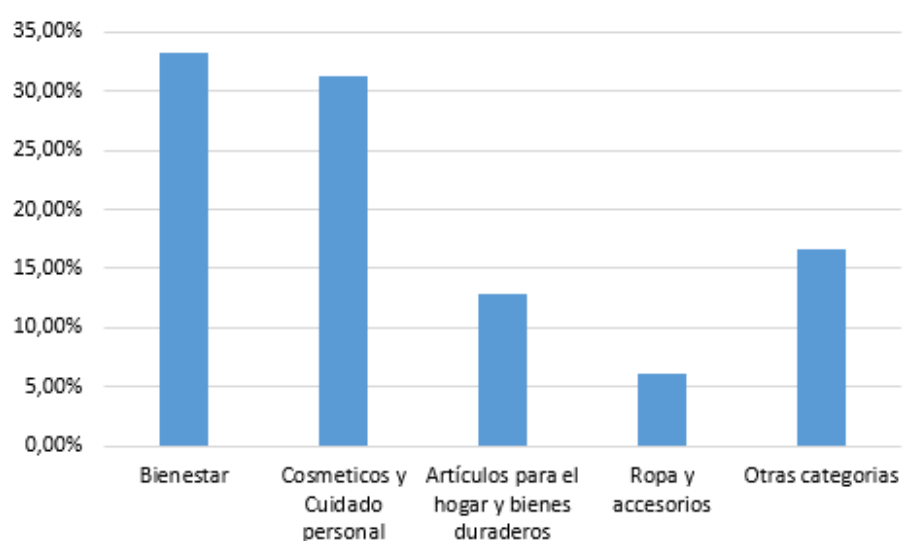

Figura 1: Composición mundial por rubro en 2018. Fuente: Datos WFDSA

La venta directa es considerada hoy en día como un modelo de negocio y es desarrollada tanto por marcas reconocidas a nivel global como por pequeñas empresas locales con perfil emprendedor para comercializar todo tipo de bienes y servicios. Por otra parte, permite a las compañías efectuar la distribución de sus productos y/o servicios a través de vendedores que tienen un comportamiento en conjunto delineado por la organización pero que individualmente poseen independencia total para decidir dónde, cuándo, a quién y cómo realizar las ventas. Este sistema permite a las organizaciones desprenderse de locales físicos donde se tendrían a disposición los productos o servicios que la misma ofrece reduciendo notablemente sus costos fijos. Por otra parte, les brinda a las personas la libertad suficiente para elegir de qué manera llevar a cabo el negocio.

Resulta notable la incidencia de la personalidad y los perfiles personales en el desempeño y rendimiento dentro de una organización; factor clave para la productividad organizacional [7]. Una de las metodologías más utilizadas en el ámbito empresarial para abordar esta temática es la metodología DISC.

El modelo inicial de la metodología fue propuesto por Marston en 1928 [8] y, en las últimas décadas, se han elaborado una gran cantidad de modificaciones, validaciones y aplicaciones que lo han convertido en una de las técnicas para crear perfiles de personalidad más populares disponibles hoy en día. Desde Clarke en 1956 [9] hasta Watson y Klassen ya en el Siglo XXI [10]. Si bien el modelo presenta un alto grado de simpleza, es una herramienta poderosa que se adapta a las necesidades de las organizaciones si se realiza un adecuado tratamiento y gestión de la información [11]. Existe una forma de mostrar la matriz DISC y es mediante el uso de palabras clave a las que se le asignan a las diferentes letras [12]. Se muestra un esquema de lo mencionado en la Figura 2.

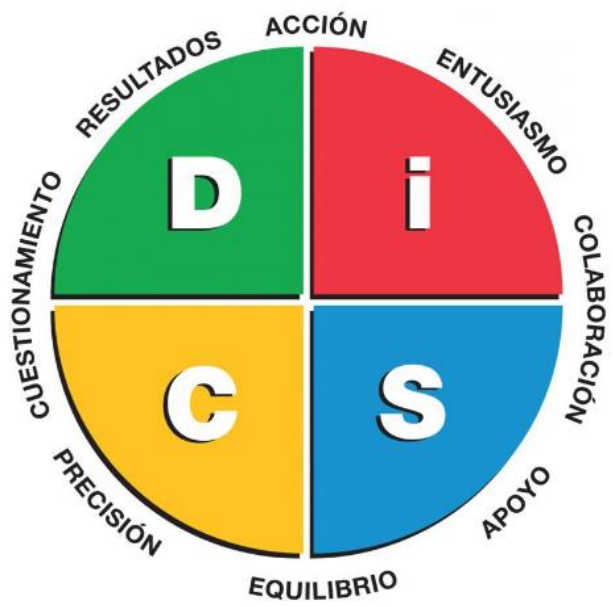

Figura 2: Matriz DISC con palabras clave. Fuente: Everything DISC.

El perfil DISC se define después de responder un cuestionario que es evaluado según cuatro factores que son aquellos que dan nombre al método. Cada una de las letras indica una serie de cualidades y comportamientos en base al estilo de personalidad que representa [13]. Tener baja una letra no es algo negativo, es solo una forma de expresar que esa persona otras características o rasgos diferentes. Las letras son: D (Dominance/Dominancia), I (Influence/Influencia), S (Stability/Estabilidad) y C (Compliance/Conformidad).

El perfil se compone del factor con más peso y el de menor peso respectivamente y se expresa con las iniciales de estos factores. Es así como una persona con un perfil DS estaría indicando un gran peso de sus características de dominancia y un bajo peso en estabilidad, dejando a la influencia y conformidad como características intermedias del individuo. Este es el parámetro con el cual se definen los rasgos de personalidad el individuo y que permite definir su perfil personal.

Por otra parte, cuando se cuenta con una gran cantidad de datos, resulta muy complejo realizar análisis que generen conocimiento, ya que estos datos no representan en sí mismos un conocimiento que aumente la competitividad o la productividad en las organizaciones [14]. En el marco del aprendizaje automático, el conjunto de métodos, algoritmos y técnicas que conforman las tareas del aprendizaje no supervisado están orientados a identificar aquella información oculta y de alto valor agregado en una o más bases de datos [15]. Una de las metodologías más utilizadas en este sentido es la Minería de Datos, que está compuesta por diversas técnicas y herramientas para la extracción de conocimiento de una determinada base de datos [16].

En este trabajo se realiza una caracterización y segmentación de vendedores que se desempeñan en modelos de negocio con sistemas de venta directa mediante técnicas de análisis cuantitativo de datos. Se estudia la fuerza de ventas, a 
través técnicas de minería de datos como son el análisis de componentes principales (PCA) y los algoritmos de clustering para identificar los grupos que comparten características y comportamientos. Para ello, se analizan datos históricos de una organización que se desarrolla en el rubro de la venta directa en Argentina hace más de 30 años y que es líder en el mercado local. A estos datos se le agregan los resultados del test DISC, el cual fue desarrollado por cada uno de los vendedores.

Con ello se pretende identificar factores clave de éxito y realizar aportes que contribuyan al diseño de estrategias empresariales, por ejemplo, planes de capacitación orientados a cierto grupo específico que necesite desarrollar aptitudes y habilidades claramente determinadas en este trabajo. El análisis toma como base el trabajo de tesis de maestría de uno de los autores del trabajo, en donde definen las variables clave del negocio, se describen estadísticamente y se realiza caracterización preliminar mediante el uso del software R. [17].

\section{CASO DE ESTUDIO}

\section{A. Objeto de estudio}

La organización que ha aportado los datos para llevar a cabo esta investigación, tiene como productos a los artículos de belleza y cuidado personal. Su misión es dar a las personas la posibilidad de desarrollarse personal, profesional y económicamente a partir de un negocio basado en sus productos. La venta se realiza por catálogo. Siempre ha aplicado el modelo de venta directa y almacena datos acerca de sus DV o directoras de ventas.

La organización tiene un esquema de redes de vendedores, donde a aquellos alcanzan un mínimo volumen de ventas se le asigna un rol de director de ventas (DV). Estos DV se abastecen directamente de la compañía y tienen su propia red de vendedores que de él o ella depende. Si uno de esos vendedores alcanza el volumen de ventas necesario para convertirse en DV, el DV del cual dependía suma una DV generada que se la llamará "Hija". A su vez, si esa "Hija" genera una DV, se le asignara a la primer DV una "Nieta" y a su vez a la "Hija" se le asignara una "Hija". Aquella DV que genere "Hijas" y "Nietas" obtendrá descuentos y aumentos en sus comisiones, incorporando a la red una opción estrategia adicional, ya que ahora no solo se puede ganar dinero por la venta en cantidades sino por la generación de hijas y nietas.

Los catálogos se renuevan bimestralmente, a este periodo de dos meses se lo denomina campaña. A cada mes contenido en esos bimestres, se lo denomina "ciclo" y dos de los mismos componen la campaña. Es crucial en la compañía el concepto de campaña porque se utiliza para la planificación de demanda, diseño de los catálogos, suministros compras y demás procesos administrativos.
Para el mercado y las compañías de la actualidad entender a las personas es una habilidad esencial a la hora de reclutar, gestionar y construir un equipo de trabajo. Tener una mirada comprensiva y objetiva de como las personalidades funcionan es una ventaja importante en el mercado y mejora sustancialmente la competitividad. Es por ello que esta información se vuelve tan relevante en este análisis, puesto que es de gran utilidad a la hora de caracterizar a las personas en el análisis sobre la situación actual. También permite tener una base de datos actualizable para aplicar métodos de predicción y gestionar el futuro de la compañía con el ingreso de nuevos y futuros vendedores.

\section{DESARROLLO}

\section{A. Datos}

La organización almacena información sobre sus DV en sus bases de datos y sistemas de gestión y es ésta la principal fuente de datos de este trabajo. Se analiza el periodo comprendido entre los años 2008 y 2018. Los datos principales a analizar son la edad, el género, los años de antigüedad, cantidad de hijas, nietas, facturación por campaña, facturación anual y el perfil de personalidad DISC. Se realiza una estructuración y un proceso ordenado de análisis, siguiendo la metodología propia de la minería de datos.

Se han considerado los datos de $178 \mathrm{DV}$, pero se excluyeron siete casos especiales detectados como valores anómalos, sobre todo desde el punto de vista de facturación y generación de descendencia [17].

Los datos incluyen información sobre 171 vendedores y contienen las siguientes variables: 1): ID (Código de identificación de cada vendedor), 2): Edad, 3): Cantidad de años activos en el periodo comprendido entre los años 20082017 (Ant.), 4): Cantidad de Hijas DV, 5): Cantidad de Nietas DV, 6): Facturación en la última Campaña (Ult.Cp.), 7): Facturación en el último ejercicio (Ult.Ej.), 8): Perfil DISC o combinación básica $(\mathrm{CB})$. Este último dato se excluye del análisis cuantitativo y se utiliza como indicador para la caracterización en el clustering. Se muestra un ejemplo de los primeros registros en la Tabla 1.

\begin{tabular}{|c|c|c|c|c|c|c|c|}
\hline ID & Edad & Ant. & Hijas & Nietas & Ult.Cp. & Ult.Ej. & CB \\
\hline 102 & 48 & 10 & 2 & 0 & $222 k$ & $1257 k$ & CI \\
\hline 108 & 45 & 10 & 0 & 0 & $144 \mathrm{k}$ & $744 \mathrm{k}$ & DC \\
\hline 109 & 55 & 10 & 1 & 1 & $206 \mathrm{k}$ & $660 \mathrm{k}$ & CD \\
\hline 113 & 57 & 10 & 3 & 0 & $116 \mathrm{k}$ & $550 \mathrm{k}$ & SD \\
\hline
\end{tabular}

Tabla 1: Ejemplo de estructuración de datos. Fuente: Elaboración propia.

Para realizar el análisis se utilizan técnicas de estadística multivariable y análisis de clúster utilizando los datos de las 171 DV. Las dos técnicas seleccionadas son la de Análisis de componentes principales (PCA) y algoritmos de clustering. 


\section{B. Análisis de componentes principales}

Con el análisis de componentes principales (PCA) se pretende generar un nuevo conjunto de variables reducido al máximo que tengan la capacidad de explicar un gran porcentaje de los datos con tan solo unas pocas componentes. Posteriormente se utilizan los resultados de esta técnica para llevar a cabo las tareas de clustering agrupando DV.

La unidad observable del PCA es el ID de cliente de cada DV. Las variables consideradas son: 1): Edad, 2): Cantidad de años como DV en el periodo 2008-2017 3): Cantidad de hijas 4): Cantidad de nietas 5): Facturación por campaña (Promedio) 6): Facturación por ejercicio (Promedio).

Para comenzar, habiendo cargado los datos en el software $\mathrm{R}$, se procede realizar el análisis de componentes principales. Este análisis debe incluir una estandarización de los datos para eliminar el problema de la magnitud de los datos y que esto no influya en el resultado del análisis.

En la Tabla 2 se muestran las desviaciones estándar y el porcentaje de representación de cada componente sobre el total.

\begin{tabular}{|c|c|c|c|c|c|c|}
\hline & PC1 & PC2 & PC3 & PC4 & PC5 & PC6 \\
\hline Desvío estándar & 1.64 & 1.14 & 1.02 & 0.67 & 0.55 & 0.45 \\
\hline Proporción & 0.45 & 0.21 & 0.17 & 0.075 & 0.05 & 0.03 \\
\hline Acumulado & 0.45 & 0.66 & 0.84 & 0.91 & 0.96 & 1.0 \\
\hline
\end{tabular}

Tabla 2: Incidencia de cada componente. Fuente: Elaboración propia.

La cantidad de componentes principales ideal para analizar este paquete de datos es de tres componentes. La elección se ve sustentada por la Tabla 2 analizada desde distintos aspectos y el criterio de Kaiser. Se puede observar que solo con la primera componente se explica casi el 50\% de los datos, el $66 \%$ considerando las dos primeras componentes $\mathrm{y}$ tomando las tres primeras un $84 \%$. El número de componentes principales a considerar debe ser determinado según su desviación estándar, según el criterio de Kaiser, porque ello representa la variabilidad. Según dicho criterio, se deben considerar aquellas componentes cuya desviación estándar sea mayor a uno. Es por ello que en este estudio se opta por tomar las 3 primeras componentes para continuar el análisis. Esto facilitara, además, la esquematización de los resultados.

Por otra parte, las componentes principales son las combinaciones lineales de las variables originales que explican la varianza de los datos. Estas están compuestas por vectores propios compuestos por un coeficiente para cada variable y se utiliza para indicar la ponderación relativa de cada variable en cada componente. En este sentido se entiende que cuanto mayor sea el valor del módulo, mayor será la incidencia de dicha variable en la componente principal. La tabla 3 permite comprender el criterio de ordenación de los datos en cada componente, considerando aquella variable que tenga mayor valor absoluto en cada una de las componentes. Además, se debe considerar la dirección de cada coeficiente, la cual está dada por el signo.

\begin{tabular}{|c|c|c|c|}
\hline & PC1 & PC2 & PC3 \\
\hline 1) & 0.2231229 & -0.70811144 & -0.1683899 \\
\hline 2) & 0.3800878 & -0.38502178 & -0.4538442 \\
\hline 3$)$ & 0.4747665 & 0.01619538 & 0.4550325 \\
\hline 4$)$ & 0.3855030 & -0.16162674 & 0.6378364 \\
\hline 5$)$ & 0.4385254 & 0.48353040 & -0.2038692 \\
\hline 6$)$ & 0.4893149 & 0.30024868 & -0.3319907 \\
\hline
\end{tabular}

Tabla 3: Criterio de ordenación. Fuente: Elaboración propia.

Si se analiza caso por caso, es en la primera componente donde se evidencia que todas las variables tienen asociaciones positivas. Esto significa que todas ordenan la componente en el mismo sentido. Se podría afirmar que esta componente mide principalmente el rendimiento de ventas y facturación de cada DV. Ya que es esa variable facturación la que posee mayor valor absoluto. $\mathrm{Al}$ analizar la segunda componente se observa que el criterio de ordenación está dado en gran medida por la edad (-). Si se considera que la antigüedad (-) también es negativa, se podría afirmar que las asociaciones negativas en esta segunda componente explican cuestiones de temporalidad. Por el contrario, las asociaciones positivas están relacionadas a la venta y la facturación (+).

En el caso de la tercera componente los datos son ordenados según la cantidad de nietas $(+)$, seguido por la cantidad de hijas (+), lo cual puede interpretarse como una componente que mide los niveles de generación de descendencia. Además, cabe señalar que una variable relevante es la antigüedad (+) que desplaza positivamente a la componente.

\section{Clustering}

Las técnicas implicadas para realizar estas tareas son: método de Elbow para definir la cantidad optima de cluster, el algoritmo de K-means para realizar los agrupamientos y el coeficiente de Silhouette y el método de inercias para validar los resultados. Además, se utiliza la combinación básica del test DISC para etiquetar a cada DV y favorecer de esta manera la interpretación de los resultados del test, combinados con el resto de los datos.

El método Elbow es un método heurístico de interpretación y validación de consistencia dentro del análisis de cluster diseñado para ayudar a encontrar el número apropiado en un conjunto de datos. El método Elbow estipula que el número óptimo de cluster es aquel en cual adicionando mayor número de los mismos no se reduce significativamente la varianza entre clusters [18]. Se observa claramente en la figura 3 que a partir de los cinco cluster la curva se vuelve similar a una recta constante, ya que existe un punto de inflexión a partir del quinto cluster. 


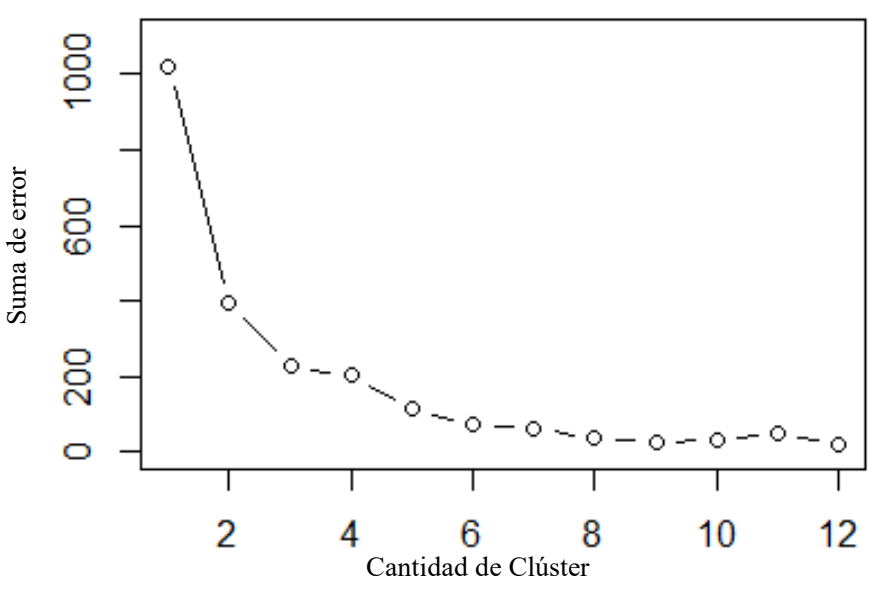

Figura 3: Método Elbow. Fuente: Elaboración propia.

En este sentido, el número óptimo de cluster podría ser cinco ya que es el mínimo número con el cual se alcanza un buen desempeño. Sin embargo, también podrían considerarse seis o más y es por ello que resulta importante validar este resultado utilizando el criterio de inercias inter-grupo, como se muestra en la Figura 4, e intra-grupo, en la Figura 5. Luego se muestra un resumen en la Tabla 4.

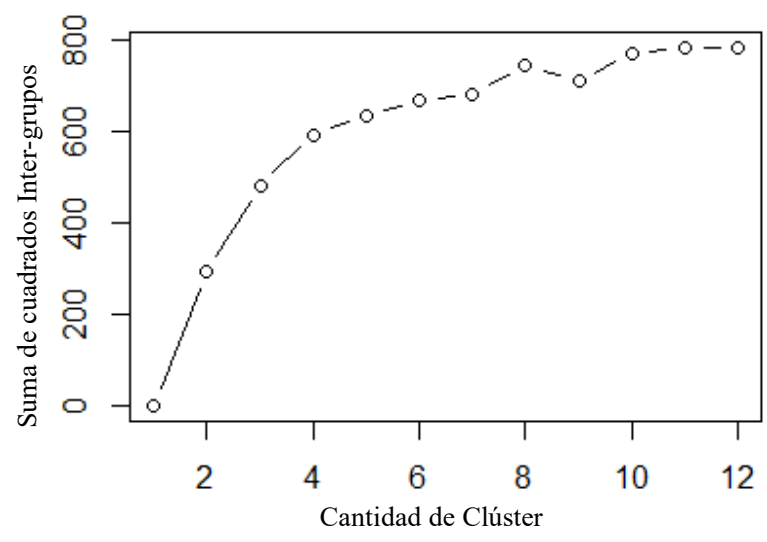

Figura 4: Inercias Inter-grupo. Fuente: Elaboración propia.

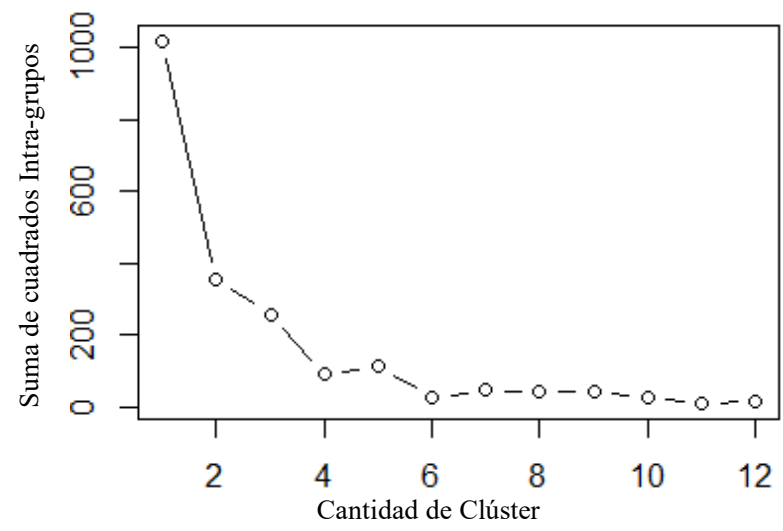

Figura 5: Inercias Intra-grupo. Fuente: Elaboración propia.

\begin{tabular}{|c|c|c|c|}
\hline $\mathrm{N}^{\circ}$ Cluster & Silhouette & Inter grupos & Intra grupos \\
\hline 3 & 0,15 & $8528779 \times 10^{\wedge} 12$ & $1423836 \times 10^{\wedge} 12$ \\
\hline 4 & 0,07 & $9043674 \times 10^{\wedge} 12$ & 908940507938 \\
\hline 5 & 0,05 & $9376086 \times 10^{\wedge} 12$ & 576528905316 \\
\hline 6 & 0,05 & $9376086 \times 10^{\wedge} 12$ & 576528905316 \\
\hline
\end{tabular}

Tabla 4: Comparación cluster óptimo. Fuente: Elaboración propia.

Se observa que tanto en la evaluación Inter-grupos como en la Intra-grupos se obtendrían muy buenos resultados utilizando cinco cluster. Si se utilizaran seis cluster se mejoraría levemente el resultado Inter-grupos, pero no habría diferencias sustanciales en la evaluación Intra-grupos y se correría riesgos de incurrir en una clustering excesivo. Por tal motivo, se adopta como valor óptimo para el estudio la utilización de cinco cluster.

A continuación, se realiza el análisis de cluster, utilizando como datos el resultado del análisis de componentes principales realizado anteriormente. Para ello se utiliza el algoritmo de $k$-means, el cual asigna a cada unidad observable al cluster con el centroide más próximo. En la figura 6 se muestra el clustering obtenido diferenciándolos por color y en donde la etiqueta de la unidad observable es la combinación básica del test DISC.
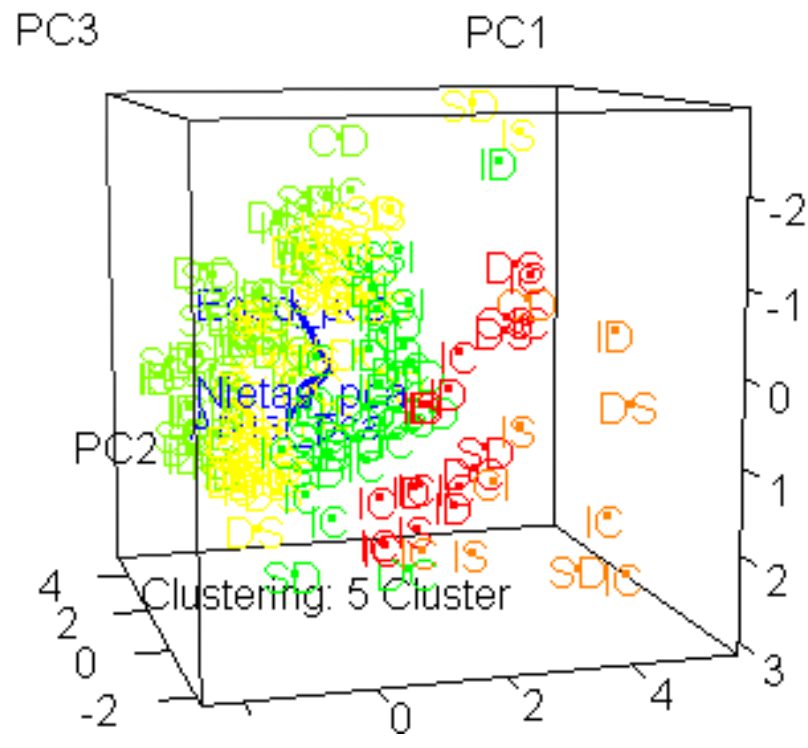

Figura 6: Clustering con PCA. Fuente: Elaboración propia.

Se pueden observar los 5 cluster diferenciados por colores y con la etiqueta de la combinación básica. El primer cluster presenta altos niveles de venta y facturación, pero niveles de descendencia significativamente bajos. Se interpreta que está compuesto por vendedores autónomos y con alto grado de independencia, ya que esto condice con sus perfiles predominantes, que son DC e IC. Su factor de éxito son su determinación, ambición y confianza.

El segundo está representado por personas jóvenes que están iniciando su carrera en el sector. Los niveles de venta 
son bajos, así como también la generación de descendencia. Por otra parte, los perfiles DISC en este grupo son diversos y, si bien no son un factor clave que caracteriza al grupo, se interpreta el mayor espectro de acciones posibles para desarrollar el talento de la fuerza de ventas y que lograr que alcancen los niveles de éxito deseados.

El tercer cluster contiene aquellos vendedores que tienen una larga trayectoria que sustenta sus logros y son referentes dentro del sistema. En este grupo se encuentran las personas con mayor antigüedad, con una edad por encima del promedio y con niveles de facturación y descendencia notables. De su perfil profesional predominante, con alta dominancia, el principal rasgo característico es la auto-motivación que los impulsa a proponerse nuevos desafíos constantemente.

En el cuarto cluster la presencia de jóvenes es nula, ya que contiene a las personas con mayor edad. Los niveles de rendimiento en ventas, facturación y descendencia son moderados y los perfiles predominantes son los SC y CD. Esto permite evidenciar que este cluster se presenta poca ambición y pareciera ser que utilizan el negocio como método de distracción y entretenimiento.

El último cluster está conformado por personas con altos niveles de ventas, facturación y descendencia. Sin embargo, pese a que el promedio de edad y antigüedad es alto, existen diversos casos con antigüedad menor a 5 años y con altos niveles de descendencia de primer nivel. Los perfiles predominantes son IC e ID. En este sentido, se considera que este grupo está conformado por personas con un marcado perfil empresarial, con resultados sumamente positivos y con gran capacidad de liderazgo y gestión del talento.

Si bien los métodos utilizados han permitido definir que el número óptimo de cluster es cinco, resulta necesario evaluar la calidad del cluster obtenido. Para ello, se calcula el coeficiente de Silhouette y además se utiliza la valuación de las inercias para concluir que este es el número de cluster apropiado para analizar este conjunto de datos. Si bien el mejor coeficiente de Silhouette lo arroja el estudio con tres cluster, tanto la inercia inter grupo como intra grupo resulta significativamente superior trabajando con 5 cluster. Por tal motivo se toma el análisis como válido para continuar con investigaciones futuras.

\section{CONCLUSIONES Y LÍNEAS FUTURAS}

En este trabajo se ha logrado caracterizar a la cartera de vendedores del caso de estudio analizado. La integración de los datos tradicionales con los datos de personalidad ha permitido que el análisis sea más completo y se puedan contrastar aspectos cuantitativos con otros cualitativos y que hacen al desarrollo personal de los vendedores. Se han identificado aquellos perfiles que más ingresos generan al sistema y cuáles son sus características referidas a su personalidad; como es el caso de los perfiles IC y DS.

Por otra parte, las técnicas de gestión de bases de datos y minería de datos utilizadas han sido eficaces a la hora de arrojar resultados que luego han sido validados cuantitativamente. En este sentido, el clustering de cinco grupos es válido y se utiliza como modelo de categorización y segmentación de la fuerza de venta e insumo para el diseño de planes de desarrollo profesional de la fuerza de ventas. Dichos planes deberían ser ad-hoc según las necesidades puntuales de cada combinación básica y a qué cluster pertenezca. De esta manera la fuerza de ventas es también beneficiaria indirecta de este análisis, debido a que la organización podrá poner a disposición capacitaciones, entrenamientos y herramientas conociendo las necesidades reales de cada grupo.

Finalmente, una de las líneas futuras de investigación está relacionada con el rendimiento de la fuerza de ventas. En este sentido, se espera realizar predicciones mediante métodos matemáticos para tener información de cómo sería el rendimiento de un vendedor actual o potencial, partiendo de la base de algunos de los datos utilizados en este trabajo. Además, otra línea en la que se pretende trabajar es en el estudio de la permanencia de cada vendedor, considerando que en este estudio se ha detectado además un punto de inflexión entre los 2 y 3 años de permanencia. Para ello también se propone utilizar técnicas de predicción, como por ejemplo redes neuronales o el método de los vecinos más cercanos.

\section{AGRADECIMIENTOS}

Agradecemos a la empresa Bagués, que ha suministrado los datos para realizar la investigación. También agradecemos a las instituciones que financian esta línea de investigación, la Universidad Nacional de Lomas de Zamora (UNLZ) a través del instrumento Lomas CyT 2019-2021, al Consejo Interuniversitario Nacional (CIN) a través de las becas EVCCIN y a la Fundación Atlántica.

\section{BIBLIOGRAFÍA}

[1] M. J. Baker, Dictionary of Marketing \& Advertising. New York: Nichols Publishing Company, 1984.

[2] P. D. Bennett, Dictionary of marketing terms. American Marketing Association, 1988.

[3] R. Ostrow and S. R. Smith, The dictionary of marketing. Fairchild Publications, 1988.

[4] R. A. Peterson and T. R. Wotruba, "What is Direct Selling? -- Definition, Perspectives, and Research Agenda," Journal of Personal Selling \& Sales Management, vol. 16, no. 4. pp. 1-16, 1996. 

vol. 19. 1990.

[6] WFDSA, "Global Sales by Product Category - 2019," Washington D.C., 2019.

[7] D. Cervone and L. A. Pervin, Personalidad: Teoría e Investigación $2^{a}$ edición. 2009.

[8] W. M. Marston, Emotions of normal people. 1928.

[9] W. V. Clarke, "The construction of an industrial selection personality test," J. Psychol., vol. 41, no. 2, pp. 379-394, Apr. 1956.

[10] R. Watson and P. Klassen, Style Insights-DISC: Instrument validation manual, vol. 28. Target Training International-Performance Systems, Ltd., Success Insights Inc: Phoenix, AZ, 2004.

[11] J. E. Tornillo, G. Pascal, J. M. Moguerza, and A. Redchuk, "Personality Traits and Business Intelligence: A Model to Improve Direct Selling Systems," in 2019 5th International Conference on Information Management (ICIM), 2019, pp. 202-206.

[12] A. Bradley, "WORKPLACE PROFILE Your Trusted Source for Everything DISC," 2018.

[13] 'Perfil DISC, 'DISC'. (Online). Disponible en: http://www.perfildisc.com," 2020.

[14] L. F. Tabares and J. F. Hernández, "Big Data Analytics : Oportunidades, Retos y Tendencias," Univ. San Buenaventura, p. 20, 2014.

[15] P.-N. Tan, M. Steinbach, and V. Kumar, Introduction to data mining. 2006.

[16] I. H. Witten, E. Frank, and M. A. Hall, Data Mining Practical Machine Learning Tools And Techniques, no. 9781447150183. 2005.

[17] J. E. Tornillo, "Estudio y Caracterización de Vendedores en Sistemas de Venta Directa en Argentina: Aplicación de Técnicas de Data Mining y Business Intelligence," Universidad Rey Juan Carlos, 2018.

[18] S. Pedro and M. Domingos, "ANÁLISE DE CLUSTERS PARA SEGMENTAÇÃO DE ESTUDANTES NUMA INSTITUIÇÃO DE ENSINO SUPERIOR,” 2019. 